\title{
Effect of Virtual Reality on Stress Reduction and Change of Physiological Parameters Including Heart Rate Variability in People With High Stress: An Open Randomized Crossover Trial
}

\begin{abstract}
Hyewon Kim ${ }^{1}$, Dong Jun Kim ${ }^{2,3}$, Seonwoo Kim ${ }^{4}$, Won Ho Chung ${ }^{5}$, Kyung-Ah Park ${ }^{6}$, James D. K. Kim ${ }^{7}$, Dowan Kim ${ }^{8}$, Min Ji Kim ${ }^{4}$, Kiwon Kim ${ }^{9}$ and Hong Jin Jeon ${ }^{2,3 *}$
\end{abstract}

OPEN ACCESS

Edited by:

Lina Gega,

University of York, United Kingdom

Reviewed by:

Siddharth Sarkar,

All India Institute of Medical

Sciences, India

Judith Amores,

Harvard Medical School,

United States

Savita G. Bhakta,

University of California, San Diego,

United States

${ }^{*}$ Correspondence:

Hong Jin Jeon

jeonhj@skku.edu

Specialty section

This article was submitted to

Public Mental Health,

a section of the journal

Frontiers in Psychiatry

Received: 06 October 2020

Accepted: 12 July 2021

Published: 10 August 2021

Citation:

Kim H, Kim DJ, Kim S, Chung WH, Park K-A, Kim JDK, Kim D, Kim MJ, Kim K and Jeon HJ (2021) Effect of Virtual Reality on Stress Reduction

and Change of Physiological

Parameters Including Heart Rate Variability in People With High Stress: An Open Randomized Crossover Trial.

Front. Psychiatry 12:614539.

doi: 10.3389/fpsyt.2021.614539
${ }^{1}$ Department of Psychiatry, Hanyang University Medical Center, Seoul, South Korea, ${ }^{2}$ Department of Psychiatry, Depression Center, Samsung Medical Center, Sungkyunkwan University School of Medicine, Seoul, South Korea, ${ }^{3}$ Department of Health Sciences and Technology, Department of Medical Device Management and Research, and Department of Clinical Research Design and Evaluation, Samsung Advanced Institute for Health Sciences and Technology (SAIHST), Sungkyunkwan University, Seoul, South Korea, ${ }^{4}$ Statistics and Data Center, Research Institute for Future Medicine, Samsung Medical Center, Seoul, South Korea, ${ }^{5}$ Department of Otorhinolaryngology, Samsung Medical Center, Sungkyunkwan University School of Medicine, Seoul, South Korea, ${ }^{6}$ Department of Ophthalmology, Samsung Medical Center, Sungkyunkwan University School of Medicine, Seoul, South Korea, ' AR Lab, Samsung Research, Samsung Electronics Co., Ltd, Seoul, South Korea, ${ }^{8}$ Advanced Solution Team, Samsung Research, Samsung Electronics Co., Ltd, Seoul, South Korea,

${ }^{9}$ Department of Psychiatry, Kangdong Sacred Heart Hospital, Hallym University College of Medicine, Seoul, South Korea

Introduction: Although, attempts to apply virtual reality (VR) in mental healthcare are rapidly increasing, it is still unclear whether VR relaxation can reduce stress more than conventional biofeedback.

Methods: Participants consisted of 83 healthy adult volunteers with high stress, which was defined as a score of 20 or more on the Perceived Stress Scale-10 (PSS-10). This study used an open, randomized, crossover design with baseline, stress, and relaxation phases. During the stress phase, participants experienced an intentionally generated shaking VR and serial-7 subtraction. For the relaxation phase, participants underwent a randomly assigned relaxation session on day 1 among VR relaxation and biofeedack, and the other type of relaxation session was applied on day 2. We compared the StateTrait Anxiety Inventory-X1 (STAI-X1), STAI-X2, the Numeric Rating Scale (NRS), and physiological parameters including heart rate variability (HRV) indexes in the stress and relaxation phases.

Results: A total of 74 participants were included in the analyses. The median age of participants was 39 years, STAI-X1 was 47.27 (SD = 9.92), and NRS was 55.51 $(\mathrm{SD}=24.48)$ at baseline. VR and biofeedback significantly decreased STAI-X1 and NRS from the stress phase to the relaxation phase, while the difference of effect between VR and biofeedback was not significant. However, there was a significant difference in electromyography, LF/HF ratio, LF total, and NN50 between VR relaxation and biofeedback. 
Conclusion: VR relaxation was effective in reducing subjectively reported stress in individuals with high stress.

Keywords: virtual reality, biofeedback, heart rate variability, stres, stress reduction

\section{INTRODUCTION}

The stress-vulnerability model proposes that, depending on the intensity of the elicited stress and the threshold for tolerating it, i.e., one's vulnerability, a stressful crisis may be contained homeostatically or may lead to psychiatric disorders (1). This model has been useful for identifying and managing psychiatric disorders, and many studies have examined how stress affects the brain and what traits of individuals affect vulnerability. The hypothalamo-pituitary-adrenal (HPA) axis has been suggested as being associated with the development of major psychiatric disorders, such as depression, mania, psychosis, and anxiety disorders (2-4). Stress increases the level of cortisol, and persistent hypercortisolemia leads to glucocorticoid receptor tolerance (5). This change also affects the hippocampus, a brain region rich in corticosteroid receptors (6). Hippocampal dysfunction can lead to inappropriate emotional responses (7), and changes of hippocampal volume have been reported in psychiatric disorders, including schizophrenia, posttraumatic stress disorder, borderline personality disorder, and depression (8-11).

Personality traits are known to explain an individual's responsiveness to stress and vulnerability (12-14). The factor of neuroticism was confirmed to have a relationship with stressrelated psychopathologies in many studies (15-18), and in particular, high anxiety is known to be a critical risk factor of hyper-responsiveness to stress and of vulnerability to developing the psychopathologies of anxiety disorders and depression (19, 20). Considering these factors, proper management of stress in the vulnerable group with high anxiety will be important for preventing the development of psychiatric disorders.

Since the introduction of virtual reality (VR) in the 1950's, equipment related to VR systems has been gradually upgraded and made lightweight, and VR has been actively used in various fields. In the medical field, attempts have been made to use VR for diagnosis and treatment of disease. The use of VR in psychiatry seems to have an advantage in effectively educating and training patients to deal with negative emotions, such as anxiety. In a variety of studies, VR was applied to the treatment of psychiatric diseases. Since clinical studies using VR in treatment of acrophobia in the 1990's (21), randomized controlled studies have been conducted not only in patients with psychiatric disorders, including post-traumatic stress disorder (22-25), anxiety disorders (26), phobias $(27,28)$, psychotic disorders (29-31), and cognitive disorders (32), but also in people with anxiety $(33,34)$ or physical pain $(33,35,36)$, and have demonstrated the effects of VR on reduction of symptoms and improved management of diseases.

There have been several studies investigating the effect of VR on stress reduction. A study using a mobile application that delivers VR showed that stress level was reduced in a costeffective and accessible manner by VR (37). Another VR study showed objective and subjective effects of relaxation by means of VR with natural scenes compared to scenes with indoor settings (38). In a work population with high stress, when immersive natural scenarios were applied to learning specific relaxation techniques, there was a reduction in chronic trait anxiety and an increase in coping skills (39). A study tried mindfulness using VR and showed the possibility of mindful attention and relaxation by means of VR (40).

Biofeedback provides non-invasive, effective psychophysiological intervention for various psychiatric disorders and is widely used in clinical settings (41). According to a meta-analysis, biofeedback training was effective in reducing self-reported stress and anxiety (42), and such a result was repeated in healthy people with high stress (43). A previous study tried to compare the relaxing effect of VR and biofeedback among healthy participants, where four relaxing treatments were made by combining display type (VR vs. computer screen) and biofeedback (electrodermal activity biofeedback vs. no biofeedback) and applied randomly to subjects, but there was no treatment-specific difference in subjective stress or physiological arousal (44).

As the Research Domain Criteria (RDoC) was developed by the National Institute of Mental Health, the need for alternatives to the traditional psychiatric nosology, such as a dimensional system in which classification is derived inductively, has emerged. As important transdiagnostic biomarkers, physiologic parameters can be used to measure physical responses to induced stress. Stress increases one's arousal and leads to various bodily responses, such as an accelerated heart rate, pupil dilatation, increased galvanic skin response, increased finger-pulse volume, and increased electromyography (EMG) activity (45). In addition, although fluctuation in the beatby-beat heart period is an intrinsic characteristic of cardiac functioning, heart rate variability (HRV) represents the ability of the heart to respond to physiological and environmental stimuli (46). Previous studies have shown the possibility of HRV as a psychophysiological measurement for reactivity to stress (47-50). For example, increased stress was associated with decreased interbeat interval (IBI) and increased the low-frequency band/the high-frequency band ratio (LF/HF ratio) (51), and anxiety was associated with decreased root mean square of the successive differences (RMSSD) (52).

In this study we aimed to identify the stress-reduction effect of VR relaxation compared to that from biofeedback after exposure to stress in people with high stress, as measured by psychological scales and physiological parameters, including HRV indexes. We tried to examine the following hypotheses in this study: 


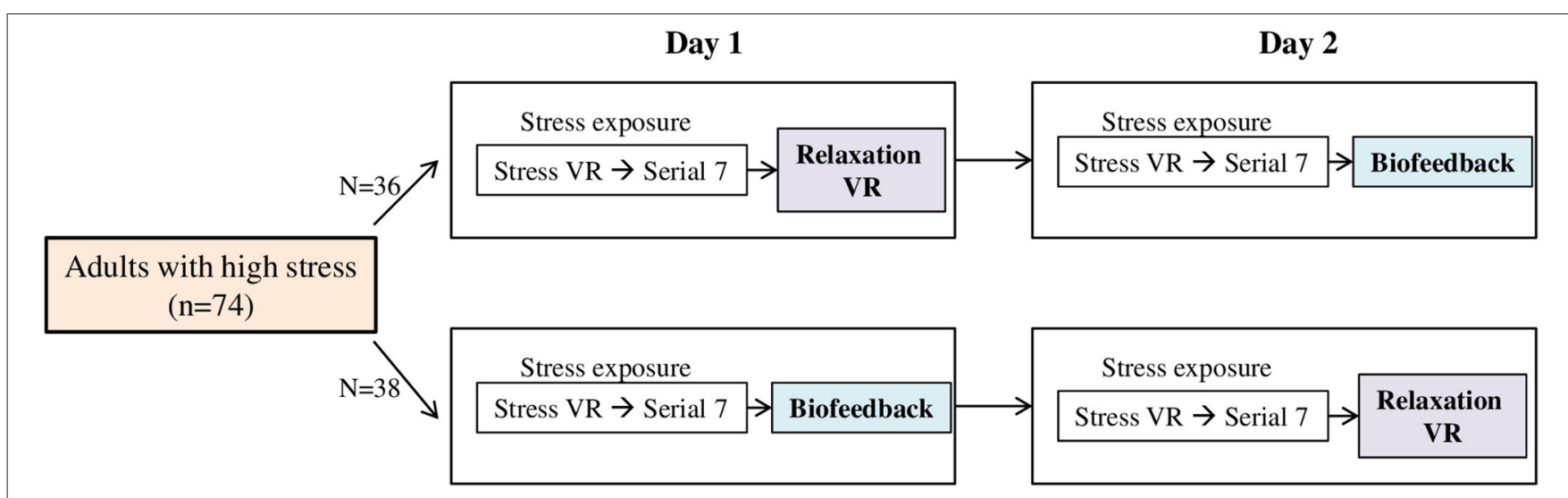

FIGURE 1 | Study design. VR, virtual reality.

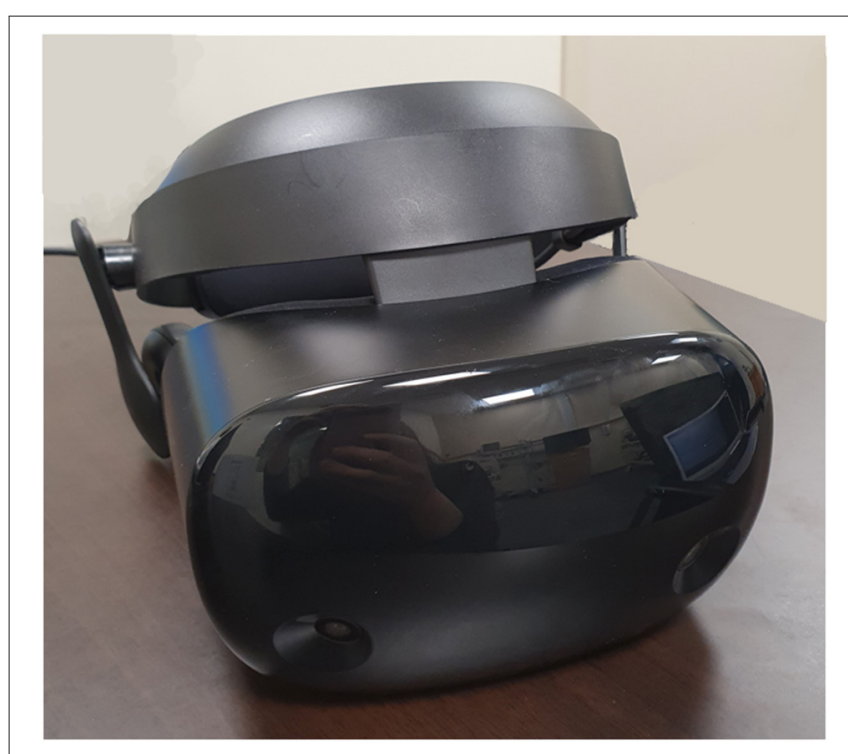

FIGURE 2 | VR device. VR, virtual reality.

(1) After exposure to stress, the effect of stress reduction in a high-stress group will be greater in a relaxation session by VR than in one by biofeedback.

(2) Change of stress after a relaxation session will be measurable by means of physiological parameters, including HRV indexes.

\section{METHODS}

\section{Participants}

We recruited 83 healthy adult volunteers with high stress, age 19 or more, from October 2016 to January 2018. We defined high stress as a score of 20 or more on the Perceived Stress Scale-10 (PSS-10) (53). Inclusion criteria were healthy persons who voluntarily participated in this study and who had no problem in understanding the study procedures and controlling the VR equipment. Those who had major psychiatric disorders, suicidal risk, neurological illnesses, including stroke or epilepsy, or serious medical illnesses were excluded. In addition, those who had medical or surgical history of psychiatric, otologic, or ophthalmologic disorders or problems with neck movements were also excluded. All participants were drug-naïve when a sample measurement was done at the baseline evaluation. At the baseline screening visit, participants were evaluated by a psychiatrist (HJJ). A psychologist who specialized in this psychiatric evaluation administered the Korean version of the Mini International Neuropsychiatric Interviews (MINI) (54) according to the Diagnostic and Statistical Manual of Mental Disorders (DSM5) (55) to the subjects to evaluate psychiatric disorders. Our study was approved by the Institutional Review Board of the Samsung Medical Center (IRB No. SMC 2016-10-007-004), and all participants gave written informed consent at enrollment in the study.

\section{Study Procedure Study Design}

This study used an open, randomized, two-period, two-treatment crossover design. The study process consisted of a baseline phase, stress phase, and relaxation phase, and was aimed to compare the differences of subjective stress reduction and physiological parameters when VR or biofeedback was applied in the relaxation phase. On day 1, participants underwent a randomly assigned relaxation session. On day 2 , the same process was conducted in the stress phase, and the other type of relaxation session was applied in the relaxation phase. We compared the differences in stress reduction and physiological parameters according to the type of relaxation sessions for participants (Figure 1). All research was conducted in a room that was exclusively prepared to block outside noise in the Clinical Trial Center located in Samsung Medical Center. Samsung Gear VR (Samsung Electronics Co., Ltd., Suwon, South Korea) was used in the stressexposure phase and relaxation phase, and the head-mounted display (HMD) device included separate screens for each eye, integrated head tracking, and stereo earphones (Figures 2, 3). 


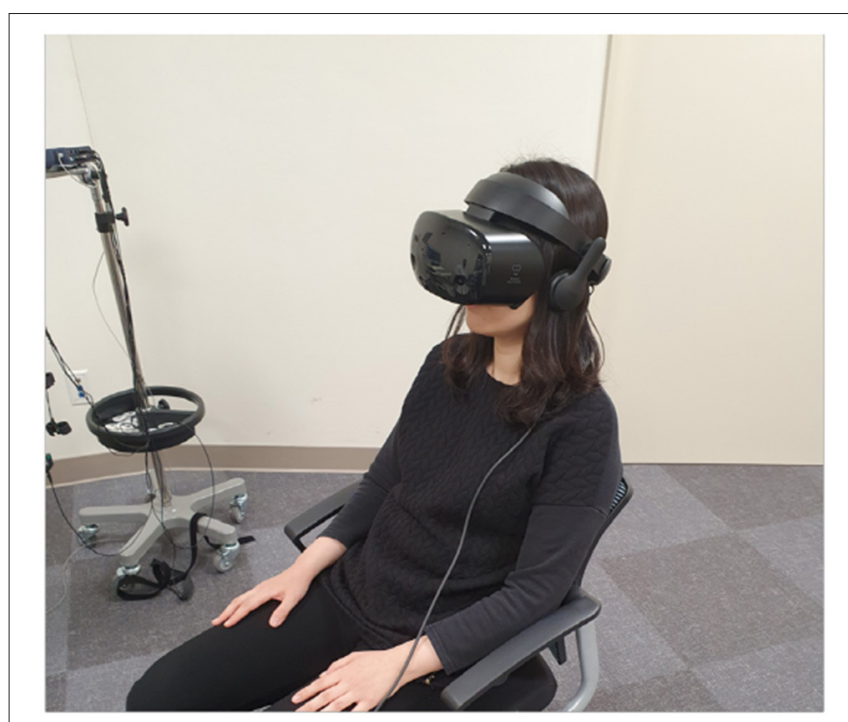

FIGURE 3 | Snapshot of VR relaxation session. VR, virtual reality.

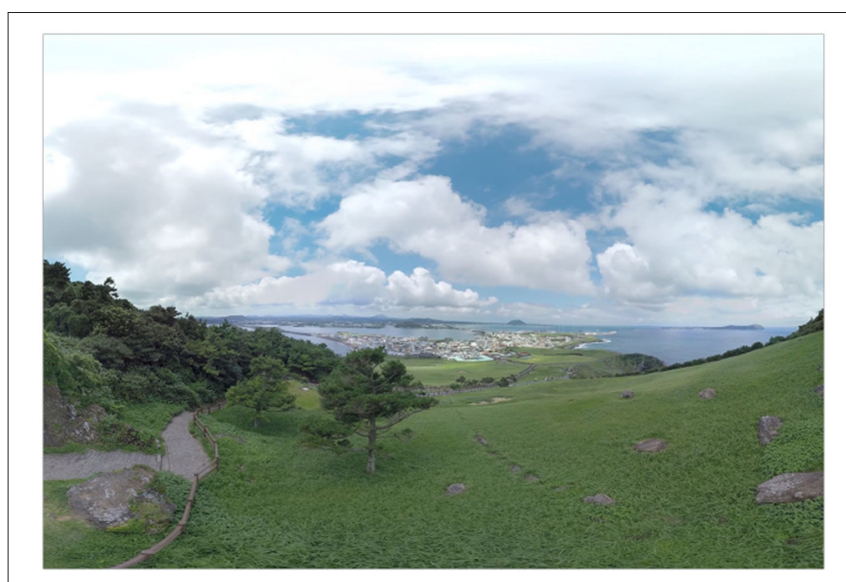

FIGURE 4 | Immersive VR video. VR, virtual reality.

\section{Stress Exposure}

In order to apply stress in a limited laboratory environment during a short time, we operationally set a protocol to induce stress, in which dizziness and discomfort were induced by experiencing VR videos with a high degree of movement, and cognitive load was given by serial subtraction. Symptoms of motion sickness such as dizziness and discomfort caused by visual-vestibular mismatch elicited stress-related physiological changes including activation of the HPA axis and elevation in level of cortisol $(56,57)$. In addition, a previous study suggested serial subtraction as an effective task in inducing a stress response as the cerebral blood flow changed and the level of salivary cortisol increased to reach a peak after the end of the task (58). First, participants experienced an intentionally generated shaking VR. The original video was provided by the Korea Land and Geospatial Informatix Corporation. The video was artificially modified for this study by adding a roll swing of a sine waveform of $30 \mathrm{~Hz}$ in the $z$-axis direction with $0.008^{\circ} / \mathrm{s}$ for each grade and image movements of 0.3 and $0.38^{\circ} \%$ s. The quantitative degree of movement of the VR video was set by referring to previous studies to sufficiently induce dizziness and discomfort in the subjects $(59,60)$. Participants were exposed to a VR video that walked on a shaky path for $3 \mathrm{~min}$ and $30 \mathrm{~s}$, and after a break of $3 \mathrm{~min}$ and $30 \mathrm{~s}$, they were exposed to a VR video once again that differed in the intensity of shaking from the first video. During the exposure to stress VR video, participants were asked to count the number of persons who appeared in the video in order to increase their attention to the video. After exposure to the stress VR video, a break of $3 \mathrm{~min}$ and $30 \mathrm{~s}$ was taken, after which the participants were asked to perform serial-7 subtraction for another $3 \mathrm{~min}$ and $30 \mathrm{~s}$ in order to be cognitively loaded.

\section{Relaxation Sessions}

Following the stress phase, we carried out relaxation sessions. On day 1, participants were exposed to VR relaxation or biofeedback as randomly assigned. On day 2, after the same process of stress exposure, the other type of relaxation session was applied as a crossover design. Both relaxation sessions using VR or biofeedback lasted for $10 \mathrm{~min}$ and $30 \mathrm{~s}$.

For the relaxation session using VR, the same device used in the stress phase, Samsung Gear VR (Samsung Electronics Co., Ltd., Suwon, South Korea), was used. During the session, participants were shown a VR video of immersive natural scenes while walking on a trekking course with famous scenery and with a relaxing soundtrack (Figure 4).

For the relaxation session using biofeedback, a computerized biofeedback system, ProComp Infiniti (Thought Technology, Ltd., Montreal, Canada), was used. We acquired information related to EMG, skin conductance, skin temperature, respiration, and heart rate/blood vessel pressure (HR/BVP) through sensors attached to the subject's body. Before the session, subjects learned relaxation reactions such as the decrease in EMG and skin conductance and increase in temperature, and were instructed to induce relaxation while watching these signals displayed on the screen during the session. In addition, the experimenter gave feedback to the participants when the parameters increased or decreased by 10\% (Figure 5).

\section{Psychological Evaluation}

At baseline, participants were evaluated by the Korean version of psychological scales including the State-Trait Anxiety InventoryX1 (STAI-X1), STAI-X2 (61), 0-100 Numeric Rating Scale (NRS) (62), Positive Affect and Negative Affect Schedule (PANAS) (63), Sheehan's Disability Scale (SDS) (64), and the five-level version of the EQ-5D (EQ-5D-5L) (65). STAI-X1 and NRS were measured repeatedly in each phase.

\section{Acquisition of Physiological Parameters}

Throughout the entire study, we acquired physiological parameters by means of sensors attached to the subject's body using the biofeedback device, ProComp Infiniti (Thought Technology, Ltd., Montreal, Canada). In addition to biofeedback parameters, HRV parameters, such as HR from inter-beat interval (IBI), mean power of the very-low-frequency band 


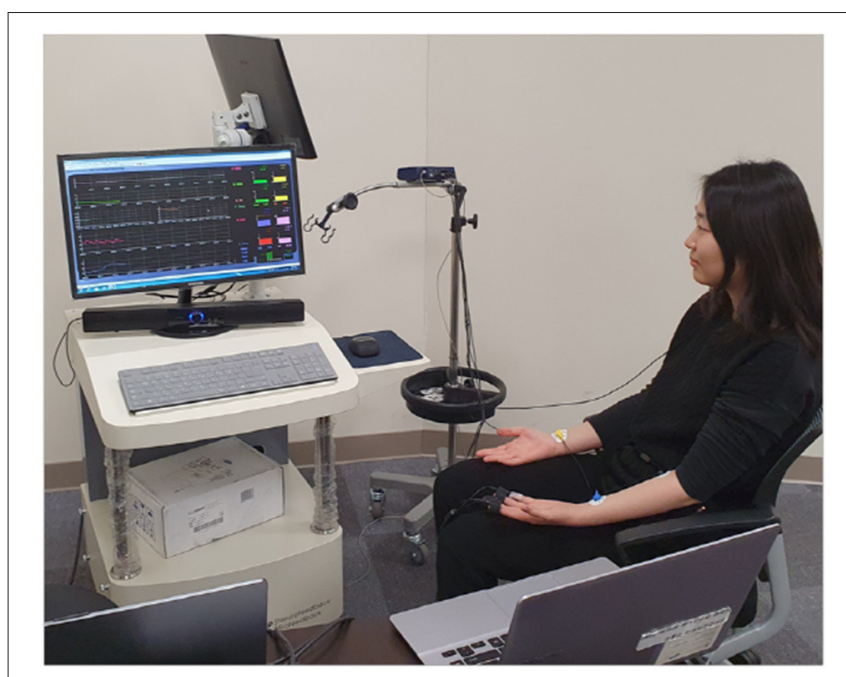

FIGURE 5 | Snapshot of biofeedback session.

(VLF: $0.0033-0.04 \mathrm{~Hz}$ ), mean power of the low-frequency band (LF: $0.04-0.15 \mathrm{~Hz}$ ), mean power of the high-frequency band (HF: $0.15-0.4 \mathrm{~Hz}$ ), ratio of $\mathrm{LF}$ to $\mathrm{HF}$ power (LF/HF ratio), number of interval differences of successive normal-to-normal $(\mathrm{NN})$ intervals greater than $50 \mathrm{~ms}$ (NN50), percentage of NN50 (pNN50) standard deviation of NN (SDNN), and RMSSD were acquired. We included parameters extracted by monitoring for $3 \mathrm{~min}$ and $30 \mathrm{~s}$ after each phase in the analyses.

\section{Simulator Sickness Questionnaire}

We repeatedly measured the simulator sickness questionnaire (SSQ) (66) before and after the experience of intentionally generated shaking VR during the stress exposure session to evaluate sickness symptoms that may occur. SSQ consists of three subscales (nausea, oculomotor, and orientation), and a higher score means more severe symptoms.

\section{Outcomes}

As the primary outcomes, we examined the change of anxiety or discomfort from the stress phase to the relaxation phase by means of psychological scales and physiological parameters, and compared the relaxation effect of VR and biofeedback.

There are individual differences in adaptation to VR equipment and satisfaction with immersion, presence, and interactivity experienced in a virtual environment (67). Moreover, sickness symptoms that may occur during VR applications can be an obstacle for relaxation through VR (68). Therefore, we defined responders as those who reported decreased NRS after VR relaxation and biofeedback compared to each stress exposure session. Among them, we compared the changes of physiological parameters during a VR session with those during a biofeedback session. In addition, we investigated the changes of SSQ before and after VR application in both responders and non-responders.
TABLE 1 | Baseline demographic and clinical characteristics of participants $(n=74)$.

\begin{tabular}{lc} 
Demographic characteristics & No or median (range) \\
\hline Age (years) & $39(19-59)$ \\
Sex (male/female) & $37 / 37$ \\
Education (years) & $16(6-22)$ \\
BMl (kg/m²) & $23(17-33)$ \\
Smokers/non-smokers & $57 / 17$ \\
Alcohol consumption (bottles/week) & $1.0(0-9)$ \\
\hline Clinical characteristics & Mean (SD) \\
\hline STAI-X1 & $47.27(9.92)$ \\
NRS & $55.51(24.48)$ \\
PSS-10 & $26.09(4.64)$ \\
PANAS & $22.26(9.82)$ \\
SDS & $15.66(6.59)$ \\
STAI-X2 & $48.16(9.88)$ \\
EQ-5D-5L & $6.47(1.63)$
\end{tabular}

BMI, Body mass index; STAI, State-Trait Anxiety Inventory; NRS, Numeric Rating Scale; PSS-10, Perceived Stress Scale; PANAS, Positive and Negative Affect Schedule; SDS, Sheehan Disability Scale; EQ-5D-5L, Five-level version of EQ-5D.

\section{Statistical Analyses}

We examined the distribution of demographic and clinical characteristics of participants. In each phase, we examined the distributions of STAI-X1, NRS, and physiological parameters. We used paired $t$-tests and Wilcoxon signed-rank tests to compare the differences of scales and physiological parameters between the stress phase and the relaxation phase according to the type of relaxation session. All statistical analyses were done with SAS version 9.4 (SAS Institute, Cary, NC, USA).

\section{RESULTS}

\section{Baseline Demographic and Clinical Characteristics of Participants}

Table 1 shows the demographic characteristics and baseline psychological evaluation data of the participants. Of the 83 enrolled subjects, two were lost to follow-up, seven were excluded from analysis because of data-extraction errors, and a total of 74 were included in the analysis. The median age of the participants was 39 years, with 37 men and women each. The mean baseline of STAI-X1 was 47.27 (SD = 9.92), and the mean baseline NRS was $55.51(\mathrm{SD}=24.48)$.

\section{Changes of STAI-X1 and NRS According to the Type of Relaxation Session}

Table 2 shows STAI-X1 and NRS at the baseline, stress, and relaxation phases when the relaxation session was applied by VR or biofeedback. The higher score on STAI-X1 indicated the more anxious state of a subject. When relaxation was induced with VR, it increased slightly from baseline 43.93 (SD = 10.05) to $45.22(\mathrm{SD}=12.27)$ after stress exposure and decreased to $38.80(\mathrm{SD}=9.96)$ after relaxation. When relaxation was induced by biofeedback, from baseline $44.76(\mathrm{SD}=9.65)$, a similar level 
TABLE 2 | STAI-X1 and NRS for the study procedure according to the type of relaxation session $(n=74)$.

\begin{tabular}{lrl}
\hline VR & Biofeedback \\
\cline { 3 - 3 } & Mean (SD)
\end{tabular}

\section{STAI-X1}

\section{Baseline}

Stress exposure

Relaxation session

$\Delta$ (Stress exposure -

relaxation session)

NRS

Baseline

43.93 (10.05)

$45.22(12.27)$

$38.80(9.96)$

$6.42(10.03)^{\star \star \star}$

$44.76(9.65)$

44.65 (11.67)

38.80 (8.94)

$5.85(7.51)^{\star \star \star}$

0.394

47.36 (26.13)

$53.80(24.53)$

41.43 (23.74)

$12.36(20.04)^{\star \star \star \star}$
49.77 (24.76)

55.62 (24.64)

42.45 (24.39)

$13.18(18.00)^{\star * \star *}$

0.561
$\Delta$ (Stress exposure -

relaxation session)

STAl, State-Trait Anxiety Inventory; NRS, Numeric Rating Scale; VR, virtual reality.

${ }^{a}$ Wilcoxon signed-rank test.

${ }^{\star * \star} p<0.001$

was maintained at $44.65(\mathrm{SD}=11.67)$ after stress exposure and decreased to $38.80(\mathrm{SD}=8.94)$ after relaxation. The difference in STAI-X1 from the stress phase to the relaxation phase was slightly higher for VR relaxation $(M=6.42, S D=10.03)$ than for biofeedback $(M=5.85, S D=7.51)$, but the difference between VR and biofeedback was not statistically significant $(\mathrm{M}=0.57$, $\mathrm{SD}=9.58, p=0.394)$ (Supplementary Figure $\mathbf{1}$ ).

The higher score on the NRS indicated greater discomfort. When relaxation was induced with VR, it increased from baseline $47.36(\mathrm{SD}=26.13)$ to $53.80(\mathrm{SD}=24.53)$ at the stress phase and decreased to $41.43(\mathrm{SD}=23.74)$ after the relaxation session. When relaxation was induced by biofeedback, NRS increased from baseline 49.77 ( $\mathrm{SD}=24.76)$ to $55.62(\mathrm{SD}=24.64)$ after the stress phase and decreased to $42.45(\mathrm{SD}=24.39)$ after the relaxation session. The difference in NRS from the stress phase to the relaxation phase was lower for VR relaxation $(\mathrm{M}=12.36$, $S D=20.04)$ than for biofeedback $(M=13.18, S D=18.00)$, but the difference was not statistically significant $(M=-0.81$, $\mathrm{SD}=18.95, p=0.561$ ) (Supplementary Figure 2).

\section{Changes of Physiological Parameters During the Baseline, Stress Phase, and Relaxation Phase}

Table 3 compares the differences of physiological parameters from the stress phase to the relaxation phase according to the type of relaxation session. EMG decreased by $0.84(\mathrm{SD}=4.53)$ in VR relaxation and decreased by $4.41(\mathrm{SD}=20.60)$ in biofeedback, showing more decrease in biofeedback $(p=0.016)$. LF total decreased by $16.87(\mathrm{SD}=119.59)$ in VR relaxation and increased by 38.57 ( $\mathrm{SD}=266.58)$ in biofeedback $(p=0.045)$. LF/HF decreased by $0.32(\mathrm{SD}=2.43)$ in VR relaxation and increased by $2.66(\mathrm{SD}=11.93)$ in biofeedback $(p=0.022)$. NN50 increased by $69.81(\mathrm{SD}=74.66)$ in VR relaxation and increased by 50.74

TABLE 3 | Changes of physiological parameters from the stress phase to the relaxation phase according to type of relaxation session $(n=74)$.

\begin{tabular}{|c|c|c|c|}
\hline \multirow[b]{3}{*}{$\Delta E M G$} & VR & Biofeedback & \multirow{3}{*}{$\begin{array}{c}\boldsymbol{p}^{\mathrm{a}} \\
0.016\end{array}$} \\
\hline & \multicolumn{2}{|c|}{ Mean (SD) } & \\
\hline & $0.84(4.53)^{\star \star}$ & $4.41(20.60)^{\star \star \star}$ & \\
\hline$\Delta$ Skin conductance & $0.43(0.65)^{\star \star \star}$ & $0.51(0.72)^{\star \star \star}$ & 0.194 \\
\hline$\Delta$ Temperature & $-0.82(1.12)^{\star \star \star}$ & $-0.80(1.04)^{\star \star \star}$ & 0.862 \\
\hline$\Delta$ Respiratory amplitude & $0.33(2.19)$ & $-0.15(2.69)$ & 0.345 \\
\hline$\Delta \mathrm{HR} / \mathrm{BVP}$ & $0.05(0.08)^{\star \star \star}$ & $0.07(0.11)^{\star \star \star}$ & 0.183 \\
\hline$\Delta \mathrm{HR}$ from $\mathrm{IBI}$ & $6.61(4.83)^{\star \star \star}$ & $5.65(4.19)^{\star \star \star}$ & 0.142 \\
\hline$\Delta \mathrm{VLF}$ total & $-2.87(70.07)$ & $-20.60(78.26)^{\star}$ & 0.091 \\
\hline$\Delta \mathrm{LF}$ total & $16.87(119.59)^{\star}$ & -38.57 (266.58) & 0.045 \\
\hline$\Delta \mathrm{HF}$ total & $68.73(148.40)^{\star \star}$ & 64.48 (192.99) & 0.555 \\
\hline$\Delta \mathrm{HRV}$ total & $113.25(290.96)^{\star \star}$ & 33.42 (485.16) & 0.054 \\
\hline$\Delta \mathrm{LF} / \mathrm{HF}$ & $0.32(2.43)$ & $-2.66(11.93)$ & 0.022 \\
\hline$\Delta \mathrm{EKG} \mathrm{IBI}$ & $-80.37(63.90)^{\star \star \star}$ & $-74.46(65.41)^{\star \star \star}$ & 0.140 \\
\hline$\Delta \mathrm{NN} 50$ & $-69.81(74.66)^{\star \star \star}$ & $-50.74(53.96)^{\star \star \star}$ & 0.021 \\
\hline$\Delta \mathrm{pNN50}$ & $-7.09(7.67)^{\star \star \star}$ & $-5.24(5.61)^{\star \star \star}$ & 0.017 \\
\hline$\triangle \mathrm{SDNN}$ & $16.83(60.24)$ & $21.41(50.67)^{\star \star}$ & 0.595 \\
\hline$\Delta \mathrm{RMSSD}$ & 15.09 (59.58) & $20.94(50.73)^{\star \star}$ & 0.892 \\
\hline
\end{tabular}

VR, virtual reality; EMG, electromyography; HR/BVP, heart rate/blood vessel pressure; $I B I$, inter-beat interval; VLF, very-low-frequency band; $L F$, low-frequency band; HF, highfrequency band; NN50, number of interval differences of successive normal-to-normal (NN) intervals > $50 \mathrm{~ms}$; $p N N 50$, percentage of NN50; SDNN, standard deviation of NN; RMSSD, the root mean square of the successive differences.

a The two groups were compared with the paired t-test when the normality assumption was satisfied and the Wilcoxon signed-rank test if not.

${ }^{*} p<0.05,{ }^{* *} p<0.01,{ }^{* \star *} p<0.001$.

$(\mathrm{SD}=53.96)$ in biofeedback $(p=0.021)$ (Supplementary Table 1 and Supplementary Table 2).

\section{Changes of Physiological Parameters According to the Type of Relaxation Session in the Responders}

We defined the responders as the subjects with decreased NRS after VR relaxation and biofeedback compared to each stress exposure session and identified the changes in their physiological parameters before and after relaxation sessions. Among a total of 39 responders, EMG showed an average decrease of 0.80 $(\mathrm{SD}=4.62)$ in VR relaxation, and an average decrease of 6.26 $(\mathrm{SD}=27.04)$ in biofeedback $(\mathrm{M}=-5.46, \mathrm{SD}=27.37, p=0.044)$. NN50 increased by $81.15(\mathrm{SD}=84.63)$ in VR relaxation and increased by $52.28(\mathrm{SD}=53.59)$ in biofeedback $(\mathrm{M}=-28.87$, $\mathrm{SD}=72.94, p=0.020$ ) (Table 4).

\section{Changes of Simulator Sickness Questionnaire Scores Before and After VR Application}

Table 5 shows the changes of SSQ before and after VR applications during stress phase. Among all subjects, total SSQ score increased significantly from $23.55($ SD $=26.03)$ to 41.30 ( $\mathrm{SD}=45.69$ ). In responders, there was no significant difference in SSQ scores at baseline and after VR application. 
TABLE 4 | Changes of physiological parameters from the stress phase to the relaxation phase according to the type of relaxation session in the responders ${ }^{a}$ $(n=39)$.

\begin{tabular}{|c|c|c|c|}
\hline & VR & Biofeedback & $\boldsymbol{p}^{\mathrm{b}}$ \\
\hline$\triangle E M G$ & $0.80(4.62)^{\star}$ & $6.26(27.04)^{\star \star \star}$ & 0.044 \\
\hline$\Delta$ Skin conductance & $0.47(0.68)^{\star \star \star}$ & $0.58(0.88)^{\star \star \star}$ & 0.178 \\
\hline$\Delta$ Temperature & $-0.91(1.01)^{\star \star \star}$ & $-0.95(1.05)^{\star \star \star}$ & 0.869 \\
\hline$\Delta$ Respiratory amplitude & $0.27(2.37)$ & $-0.08(2.04)$ & 0.330 \\
\hline$\Delta \mathrm{HR} / \mathrm{BVP}$ & $0.04(0.06)^{\star \star \star}$ & $0.07(0.13)^{\star \star \star}$ & 0.153 \\
\hline$\Delta \mathrm{HR}$ from IBI & $7.00(5.45)^{\star \star \star}$ & $5.83(4.96)^{\star \star \star}$ & 0.379 \\
\hline$\Delta \mathrm{VLF}$ total & -12.06 (69.55) & -26.85 (89.59) & 0.359 \\
\hline$\Delta L F$ total & $-6.80(115.16)$ & -83.44 (332.79) & 0.288 \\
\hline$\Delta \mathrm{HF}$ total & 50.85 (148.92) & 78.41 (200.61) & 0.349 \\
\hline$\Delta \mathrm{HRV}$ total & $63.04(269.08)$ & $1.11(560.19)$ & 0.328 \\
\hline$\Delta \mathrm{LF} / \mathrm{HF}$ & -0.07 (2.39) & $-4.37(15.24)$ & 0.081 \\
\hline$\Delta E K G|B|$ & $-87.16(77.43)^{\star \star \star}$ & $-84.93(80.32)^{\star \star \star}$ & 0.342 \\
\hline$\Delta \mathrm{NN} 50$ & $-81.15(84.63)^{\star \star \star}$ & $-52.28(53.59)^{\star \star \star}$ & 0.020 \\
\hline$\Delta \mathrm{pNN} 50$ & $-8.19(8.55)^{\star \star \star}$ & $-5.33(5.36)^{\star \star \star}$ & 0.020 \\
\hline$\triangle \mathrm{SDNN}$ & 1.67 (58.33) & $26.72(53.48)^{\star}$ & 0.116 \\
\hline$\Delta \mathrm{RMSSD}$ & $-0.22(57.28)$ & $24.89(51.69)^{\star}$ & 0.092 \\
\hline
\end{tabular}

VR, virtual reality; EMG, electromyography; HR/BVP, heart rate/blood vessel pressure; $I B I$, inter-beat interval; VLF, very-low-frequency band; $L F$, low-frequency band; $H F$, highfrequency band; NN50, pNN50, percentage of NN50; number of interval differences of successive normal-to-normal (NN) intervals > $50 \mathrm{~ms}$; SDNN, standard deviation of NN; RMSSD, the root mean square of the successive differences.

a Subjects who reported a decrease in NRS after a relaxation session from stress phase.

${ }^{b}$ The two groups were compared with the paired t-test when the normality assumption was satisfied and the Wilcoxon signed-rank test if not.

${ }^{\star} p<0.05,{ }^{* \star *} p<0.001$.

In non-responders, the baseline total score increased from 21.25 $(\mathrm{SD}=22.88)$ to $47.25(\mathrm{SD}=52.97)$ after VR application, which was larger than the average of all subjects.

\section{DISCUSSION}

In this study, when VR relaxation or biofeedback was undertaken after stress exposure in people with high stress, although both treatments reduced subjective stress significantly, the difference in subjective stress reduction between the treatments was not significant. When we evaluated the change of stress by means of physiological parameters in both treatments, there was a significant difference in EMG, LF total, LF/HF ratio, and NN50. In particular, the main analysis and sensitivity analysis consistently showed a greater increase of NN50 in VR relaxation than in biofeedback and a greater decrease of EMG in biofeedback than in VR relaxation.

Although, VR was effective in subjective stress reduction in this study, the magnitude of the effect was not significantly different from that of biofeedback. This is consistent with the finding of Rockstroh et al. (44) which compared the effect of relaxation through VR and biofeedback. However, there are differences in methodology between their study and current study. They compared the electrodermal activity and 20-item state version of the STAI between subjects while applying one of four conditions disentangled according to display type (VR vs. computer screen) and biofeedback (electrodermal activity biofeedback vs. no biofeedback) to healthy participants, while we compared the effects of VR relaxation and biofeedback within individuals through a psychological scale and various physiological parameters among highly stressed subjects by crossover design. When evaluated by the subjective scales, the change of STAI-X1, measuring state anxiety, was greater in $\mathrm{VR}$, and the change of NRS, measuring subjective discomfort, was greater in biofeedback. Although, additional research is needed, these findings are presumed to be related to how VR and biofeedback induce relaxation and stress reduction. In the protocol implemented in this study, VR reduced stress by making subjects experience an immersive video showing natural settings that made them feel like they were in a calm and peaceful place, whereas, biofeedback induced relaxation by training participants to consciously affect physiological activities that are generally done unconsciously, by providing feedback on changes in heart rate, respiration rate, or skin conductance.

These differences in scales may be better explained by changes in physiologic parameters. Among the physiological parameters, the decrease in EMG was greater in biofeedback, the increase in NN50 and the decrease in LF/HF ratio were greater in VR, and each change is known to reflect relaxation or stress reduction. The EMG, the sensor of superficial EMG, reflects the degree of muscle tension, which decreases upon relaxation. Therefore, the results of our study suggest that although the feedback of the EMG change was not given to participants according to the protocol, muscle relaxation was done effectively by a conscious control in physiological activities, which was more efficient than by VR relaxation. NN50 was increased more in VR relaxation than by biofeedback, and the result was consistent in both the main analysis and the sensitivity analysis. NN50, one of HRV time-domain measures (69), is closely correlated with peripheral nervous system (PNS) activity (70). Previous studies have shown that when stress is induced, the percentage of NN50 decreases $(47,71)$. Inferred from these findings, the increase in NN50 in VR relaxation suggests that VR may be more efficient for stress reduction by means of parasympathetic activity. The LF/HF ratio is used to estimate the balance between the sympathetic nervous system (SNS) and PNS, because both PNS and SNS activities contribute to LF power, and PNS activity contributes to HF power (72). A low $\mathrm{LF} / \mathrm{HF}$ ratio reflects parasympathetic dominance, whereas, a high LF/HF ratio indicates sympathetic dominance (69). Previous studies showed that an increase in the $\mathrm{LF} / \mathrm{HF}$ ratio is observed in a stress situation $(48,73)$. Therefore, our finding can be explained by the fact that parasympatheticdominant physiological responses were induced more by means of VR relaxation, and this leads to reduction of stress.

This study showed the possibility of using VR as a novel tool for stress management. It has been shown that immersion and presence in a natural setting by means of VR have an effect on relaxation, which uses the knowledge that the natural setting generally has a mentally restorative effect $(74,75)$. VR can be used as a method for providing treatment of new contents as in this study, but it could also be used to effectively deliver existing tools, such as the mindfulness approach or 
TABLE 5 | Changes of SSQ before and after stress exposure using intentionally generated shaking VR.

\begin{tabular}{|c|c|c|c|c|}
\hline & Nausea scores & Oculomotor scores & Disorientation scores & Total scores \\
\hline \multicolumn{5}{|l|}{ All subjects $(n=74)$} \\
\hline Baseline SSQ & $16.50(22.12)$ & $20.18(21.27)$ & $27.46(35.99)$ & $23.55(26.03)$ \\
\hline After VR application during stress phase & $32.36(42.83)$ & $31.86(31.01)$ & $49.66(59.73)$ & $41.30(45.69)$ \\
\hline$\Delta(\mathrm{VR} \text { application }- \text { baseline })^{\mathrm{a}}$ & $15.86(45.31)^{\star \star}$ & $11.68(32.04)^{\star \star}$ & $22.20(57.41)^{\star \star \star}$ & $17.75(45.64)^{\star \star \star}$ \\
\hline \multicolumn{5}{|l|}{ Responders $(n=39)$} \\
\hline$\Delta(\mathrm{VR} \text { application }- \text { baseline })^{\mathrm{a}}$ & $6.36(33.79)$ & $8.75(27.58)$ & $13.56(45.33)$ & $10.34(34.85)$ \\
\hline \multicolumn{5}{|l|}{ Non-responders $(n=35)$} \\
\hline Baseline SSQ & $15.26(21.36)$ & $17.76(19.01)$ & 25.06 (29.39) & $21.25(22.88)$ \\
\hline After VR application during stress phase & $41.70(49.95)$ & 32.70 (34.28) & $56.87(68.81)$ & $47.25(52.97)$ \\
\hline$\Delta(\mathrm{VR} \text { application - baseline })^{\mathrm{a}}$ & $26.44(53.97)^{\star \star}$ & $14.94(36.51)^{\star}$ & $31.82(67.82)^{\star \star}$ & $26.01(54.61)^{\star \star}$ \\
\hline
\end{tabular}

SSQ, Simulator sickness questionnaire.

apaired t-test.

${ }^{\star} p<0.05,{ }^{* *} p<0.01,{ }^{* \star *} p<0.001$.

cognitive behavioral therapy (CBT) for management of stress or anxiety. Conventional non-pharmacological interventions have the disadvantage that individual variance is large in motivation, dedication, mindedness, and capacity for conscious control of physiologic responses, all of which affects the outcome of treatment. On the other hand, the effect of individual factors can be alleviated by effectively delivering treatment contents by inducing presence and immersion using visual and auditory stimuli. In addition, the new delivery method by means of VR is more cost-effective than conventional tools, which require well-trained clinicians or therapists. With $\mathrm{VR}$, it is possible to maintain anonymity and facilitate treatment access for users.

Another important finding of this study is that in relaxation through VR, adverse events such as sickness symptoms can interfere with the relaxation process. In this study, we compared the sickness symptoms at baseline and after VR sessions in responders and non-responders. As a result, responders did not show a significant increase in sickness symptoms, whereas, non-responders showed a significant increase in all domains of sickness symptoms. Although, further research is needed, this suggests that there are characteristics of the person receiving benefits from VR relaxation, and along with physiological parameters, this may be an important factor in the selection of methods of non-pharmacological relaxation such as VR or biofeedback.

This study has several limitations. First, exposed stressors in a laboratory environment may not be delivered effectively depending on the individual. In this study, stress exposure was elicited by means of task execution in an intentionally generated shaking VR and cognitive load by means of serial-7 subtraction. We operationally set up this method to elicit stress in a short time in a limited laboratory environment. However, it is not clear whether performing these tasks could be a significant psychosocial stressor for all subjects, because a stressor can be perceived as either challenging or discomforting (76-78). That is, depending on the individual's intrinsic tolerance, the given stress may not be stressful. However, since we targeted people who are expected to have high vulnerability to stress, we expect the discomfort caused by stress exposure to be greater than in the general population. Second, the time to induce stress reduction through VR or biofeedback may not be sufficient. Each relaxation session lasted for a total of $10 \mathrm{~min}$ and $30 \mathrm{~s}$, but depending on the individual, the time may be insufficient to induce the maximum level of relaxation. Third, since this study investigated the short-term effect of stress reduction during biofeedback and VR relaxation, further, studies investigating the long-term effect of VR relaxation on stress reduction are needed. Fourth, since this study was conducted with healthy adults, it is difficult to apply the results to children or adolescents. In addition, since the oldest subject was 59 years old, it is difficult to apply our results to the elderly. Fifth, the effect of relaxation through VR or biofeedback should be interpreted in the context of the methodology of study protocol. In this study, e.g., during VR application, the subjects virtually walked without actual movement. The conditions in VR may affect presence and adverse events including cybersickness, and these can also affect relaxation. Regarding biofeedback, in addition to inducing relaxation by feeding back changes in physiological parameters, other methods such as breathing training, progressive muscle relaxation, and guided imagery can be used and the applied protocol may affect the results. In particular, considering that the content of VR relaxation was experiencing a relaxing natural scene, if guided imagery was included in the biofeedback protocol, there might be a difference in the results. Finally, we did not evaluate factors assessing immersion, presence, and interactivity during the VR experience, and these can affect the relaxation through VR.

This study has the following strengths. Although, there was no significant difference in the subjective relaxation between VR 
and biofeedback, we could identify the physiological changes according to each treatment by comparing changes in various physiological parameters between the treatments within an individual. In addition, by adopting a crossover design, it has the strength of minimizing the confounding effect that could occur due to between-subject variability. This study showed the possibility of VR as a useful tool for stress reduction. In addition to the protocol of VR relaxation applied in this study, future development of new technologies and efficient treatment tools will enable cost-effective, easily accessible, and personalized management of stress.

In conclusion, this study found that VR is effective in reducing subjective stress in people with high stress. Although the effect of VR relaxation was not significantly superior to biofeedback, it showed significant differences in several physiological parameters, and these can act as important factors that affect the selection of non-pharmacological relaxation such as VR in clinical settings.

\section{DATA AVAILABILITY STATEMENT}

The raw data supporting the conclusions of this article will be made available by the authors, without undue reservation.

\section{ETHICS STATEMENT}

The studies involving human participants were reviewed and approved by Institutional Review Board of the Samsung Medical Center. The patients/participants provided their written informed consent to participate in this study.

\section{REFERENCES}

1. Zubin J, Spring B. Vulnerability-a new view of schizophrenia. J Abnorm Psychol. (1977) 86:103-26. doi: 10.1037/0021-843X.86.2.103

2. Garner B, Pariante CM, Wood SJ, Velakoulis D, Phillips L, Soulsby B, et al. Pituitary volume predicts future transition to psychosis in individuals at ultra-high risk of developing psychosis. Biol Psychiatry. (2005) 58:41723. doi: 10.1016/j.biopsych.2005.04.018

3. Nestler EJ, Barrot M, DiLeone RJ, Eisch AJ, Gold SJ, Monteggia LM. Neurobiology of depression. Neuron. (2002) 34:1325. doi: 10.1016/S0896-6273(02)00653-0

4. Pariante CM, Vassilopoulou K, Velakoulis D, Phillips L, Soulsby B, Wood SJ, et al. Pituitary volume in psychosis. Br J Psychiatry. (2004) 185:510. doi: 10.1192/bjp.185.1.5

5. Goh C, Agius M. The stress-vulnerability model how does stress impact on mental illness at the level of the brain and what are the consequences? Psychiatr Danub. (2010) 22:198-202.

6. De Kloet ER, Vreugdenhil E, Oitzl MS, Joels M. Brain corticosteroid receptor balance in health and disease. Endocr Rev. (1998) 19:269301. doi: 10.1210/edrv.19.3.0331

7. Davidson RJ, Pizzagalli D, Nitschke JB, Putnam K. Depression: perspectives from affective neuroscience. Annu Rev Psychol. (2002) 53:545-74. doi: 10.1146/annurev.psych.53.100901.135148

8. Felmingham K, Williams LM, Whitford TJ, Falconer E, Kemp $\mathrm{AH}$, Peduto A, et al. Duration of posttraumatic stress disorder

\section{AUTHOR CONTRIBUTIONS}

HK contributed to interpreting the data and writing the original draft of the manuscript. SK and MJK contributed to statistical analyses. DJK, KK, WHC, and K-AP contributed to data collection and processing. JDKK and DK contributed to production of the virtual reality videos. WHC, K-AP, and HJJ contributed to conceptualization. HJJ contributed to project administration and supervision. All authors contributed to the writing and editing of the manuscript.

\section{FUNDING}

This research was supported by Quantification of VR (VR) related motion sickness and visual fatigue, and biofeedback by Samsung Medical Center. It was also supported by a grant of the Korea Health Technology R\&D Project through the Korea Health Industry Development Institute (KHIDI), funded by the Ministry of Health \& Welfare, Republic of Korea (No. HR21C0885) and the Bio \& Medical Technology Development Program of the NRF funded by the Korean government, MSIP (No. NRF2017M3A9F1027323; PI HJJ). The Samsung Medical Center and the National Research Foundation of Korea had no further role in the study design, in the collection, analysis, and interpretation of data, in the writing of the report, or in the decision to submit the paper for publication.

\section{SUPPLEMENTARY MATERIAL}

The Supplementary Material for this article can be found online at: https://www.frontiersin.org/articles/10.3389/fpsyt. 2021.614539/full\#supplementary-material

predicts hippocampal grey matter loss. Neuroreport. (2009) 20:1402-6. doi: 10.1097/WNR.0b013e3283300fbc

9. Sheline YI, Wang PW, Gado MH, Csernansky JG, Vannier MW. Hippocampal atrophy in recurrent major depression. Proc Natl Acad Sci U S A. (1996) 93:3908-13. doi: 10.1073/pnas.93.9.3908

10. Sumich A, Chitnis XA, Fannon DG, O'Ceallaigh S, Doku VC, Falrowicz A, et al. Temporal lobe abnormalities in first-episode psychosis. Am J Psychiatry. (2002) 159:1232-5. doi: 10.1176/appi.ajp.159.7.1232

11. Weniger G, Lange C, Sachsse U, Irle E. Reduced amygdala and hippocampus size in trauma-exposed women with borderline personality disorder and without posttraumatic stress disorder. J Psychiatry Neurosci. (2009) 34:383-8.

12. DiGangi JA, Gomez D, Mendoza L, Jason LA, Keys CB, Koenen KC. Pretrauma risk factors for posttraumatic stress disorder: a systematic review of the literature. Clin Psychol Rev. (2013) 33:728-44. doi: 10.1016/j.cpr.2013.05.002

13. Duggan C, Milton J, Egan V, McCarthy L, Palmer B, Lee A. Theories of general personality and mental disorder. Br J Psychiatry Suppl. (2003) 44:S1923. doi: 10.1192/bjp.182.44.s19

14. Tosevski DL, Milovancevic MP, Gajic SD. Personality and psychopathology of university students. Curr Opin Psychiatry. (2010) 23:48-52. doi: 10.1097/YCO.0b013e328333d625

15. Barlow DH, Ellard KK, Sauer-Zavala S, Bullis JR, Carl JR. The origins of neuroticism. Perspect Psychol Sci. (2014) 9:48196. doi: $10.1177 / 1745691614544528$ 
16. Brown TA, Rosellini AJ. The direct and interactive effects of neuroticism and life stress on the severity and longitudinal course of depressive symptoms. J Abnorm Psychol. (2011) 120:844-56. doi: 10.1037/a0023035

17. Kendler KS, Myers J. The genetic and environmental relationship between major depression and the five-factor model of personality. Psychol Med. (2010) 40:801-6. doi: 10.1017/S0033291709991140

18. Ormel J, Jeronimus BF, Kotov R, Riese H, Bos EH, Hankin B, et al. Neuroticism and common mental disorders: meaning and utility of a complex relationship. Clin Psychol Rev. (2013) 33:686-97. doi: 10.1016/j.cpr.2013.04.003

19. Rogers J, Raveendran M, Fawcett GL, Fox AS, Shelton SE, Oler JA, et al. CRHR1 genotypes, neural circuits and the diathesis for anxiety and depression. Mol Psychiatry. (2013) 18:700-7. doi: 10.1038/mp.2012.152

20. Sandi C, Richter-Levin G. From high anxiety trait to depression: a neurocognitive hypothesis. Trends Neurosci. (2009) 32:31220. doi: 10.1016/j.tins.2009.02.004

21. Rothbaum BO, Hodges LF, Kooper R, Opdyke D, Williford JS, North M. Effectiveness of computer-generated (virtual reality) graded exposure in the treatment of acrophobia. Am J Psychiatry. (1995) 152:6268. doi: 10.1176/ajp.152.4.626

22. Beidel DC, Frueh BC, Neer SM, Bowers CA, Trachik B, Uhde TW, et al. Trauma management therapy with virtual-reality augmented exposure therapy for combat-related PTSD: a randomized controlled trial. J Anxiety Disord. (2019) 61:64-74. doi: 10.1016/j.janxdis.2017.08.005

23. McLay RN, Wood DP, Webb-Murphy JA, Spira JL, Wiederhold MD, Pyne $\mathrm{JM}$, et al. A randomized, controlled trial of virtual reality-graded exposure therapy for post-traumatic stress disorder in active duty service members with combat-related post-traumatic stress disorder. Cyberpsychol Behav Soc Netw. (2011) 14:223-9. doi: 10.1089/cyber.2011.0003

24. Reger GM, Koenen-Woods P, Zetocha K, Smolenski DJ, Holloway KM, Rothbaum BO, et al. Randomized controlled trial of prolonged exposure using imaginal exposure vs. virtual reality exposure in active duty soldiers with deployment-related posttraumatic stress disorder (PTSD). J Consult Clin Psychol. (2016) 84:946-59. doi: 10.1037/ccp0000134

25. Rothbaum BO, Price M, Jovanovic T, Norrholm SD, Gerardi M, Dunlop B, et al. A randomized, double-blind evaluation of D-cycloserine or alprazolam combined with virtual reality exposure therapy for posttraumatic stress disorder in Iraq and Afghanistan War veterans. Am J Psychiatry. (2014) 171:640-8. doi: 10.1176/appi.ajp.2014.13121625

26. Bouchard S, Dumoulin S, Robillard G, Guitard T, Klinger E, Forget H, et al. Virtual reality compared with in vivo exposure in the treatment of social anxiety disorder: a three-arm randomised controlled trial. $\mathrm{Br} J$ Psychiatry. (2017) 210:276-83. doi: 10.1192/bjp.bp.116.184234

27. Diemer J, Domschke K, Muhlberger A, Winter B, Zavorotnyy M, Notzon S, et al. Acute anxiolytic effects of quetiapine during virtual reality exposure-a double-blind placebo-controlled trial in patients with specific phobia. Eur Neuropsychopharmacol. (2013) 23:1551-60. doi: 10.1016/j.euroneuro.2013.01.001

28. Shiban Y, Diemer J, Muller J, Brutting-Schick J, Pauli P, Muhlberger A. Diaphragmatic breathing during virtual reality exposure therapy for aviophobia: functional coping strategy or avoidance behavior? A pilot study. BMC Psychiatry. (2017) 17:29. doi: 10.1186/s12888-016-1181-2

29. du Sert OP, Potvin S, Lipp O, Dellazizzo L, Laurelli M, Breton R, et al. Virtual reality therapy for refractory auditory verbal hallucinations in schizophrenia: a pilot clinical trial. Schizophr Res. (2018) 197:17681. doi: 10.1016/j.schres.2018.02.031

30. Park KM, Ku J, Choi SH, Jang HJ, Park JY, Kim SI, et al. A virtual reality application in role-plays of social skills training for schizophrenia: a randomized, controlled trial. Psychiatry Res. (2011) 189:16672. doi: 10.1016/j.psychres.2011.04.003

31. Pot-Kolder R, Geraets CNW, Veling W, van Beilen M, Staring ABP, Gijsman HJ, et al. Virtual-reality-based cognitive behavioural therapy versus waiting list control for paranoid ideation and social avoidance in patients with psychotic disorders: a single-blind randomised controlled trial. Lancet Psychiatry. (2018) 5:217-26. doi: 10.1016/S2215-0366(18)30053-1

32. Doniger GM, Beeri MS, Bahar-Fuchs A, Gottlieb A, Tkachov A, Kenan H, et al. Virtual reality-based cognitive-motor training for middle-aged adults at high Alzheimer's disease risk: a randomized controlled trial. Alzheimers Dement. (2018) 4:118-29. doi: 10.1016/j.trci.2018.02.005
33. Eijlers R, Legerstee JS, Dierckx B, Staals LM, Berghmans J, van der Schroeff MP, et al. Development of a virtual reality exposure tool as psychological preparation for elective pediatric day care surgery: methodological approach for a randomized controlled trial. JMIR Res Protoc. (2017) 6:e174. doi: 10.2196/resprot.7617

34. Ryu JH, Park SJ, Park JW, Kim JW, Yoo HJ, Kim TW, et al. Randomized clinical trial of immersive virtual reality tour of the operating theatre in children before anaesthesia. Br J Surg. (2017) 104:162833. doi: 10.1002/bjs. 10684

35. Gold JI, Mahrer NE. Is virtual reality ready for prime time in the medical space? A randomized control trial of pediatric virtual reality for acute procedural pain management. J Pediatr Psychol. (2018) 43:26675. doi: 10.1093/jpepsy/jsx129

36. JahaniShoorab N, Ebrahimzadeh Zagami S, Nahvi A, Mazluom SR, Golmakani $\mathrm{N}$, Talebi $\mathrm{M}$, et al. The effect of virtual reality on pain in primiparity women during episiotomy repair: a randomize clinical trial. Iran J Med Sci. (2015) 40:219-24.

37. Gao K, Boyd C, Wiederhold MD, Wiederhold BK. VR mobile solutions for chronic stress reduction in young adults. Stud Health Technol Inform. (2014) 199:88-93. doi: 10.3233/978-1-61499-401-5-88

38. Anderson AP, Mayer MD, Fellows AM, Cowan DR, Hegel MT, Buckey JC. Relaxation with immersive natural scenes presented using virtual reality. Aerosp Med Hum Perform. (2017) 88:520-6. doi: 10.3357/AMHP.4747.2017

39. Gaggioli A, Pallavicini F, Morganti L, Serino S, Scaratti C, Briguglio M, et al. Experiential virtual scenarios with real-time monitoring (interreality) for the management of psychological stress: a block randomized controlled trial. J Med Internet Res. (2014) 16:e167. doi: 10.2196/jmir.3235

40. Cikajlo I, Cizman Staba U, Vrhovac S, Larkin F, Roddy M. A cloud-based virtual reality app for a novel telemindfulness service: rationale, design and feasibility evaluation. JMIR Res Protoc. (2017) 6:e108. doi: 10.2196/resprot.6849

41. Schoenberg PL, David AS. Biofeedback for psychiatric disorders: a systematic review. Appl Psychophysiol Biofeedback. (2014) 39:109-35. doi: 10.1007/s10484-014-9246-9

42. Goessl VC, Curtiss JE, Hofmann SG. The effect of heart rate variability biofeedback training on stress and anxiety: a meta-analysis. Psychol Med. (2017) 47:2578-86. doi: 10.1017/S0033291717001003

43. Ratanasiripong P, Park JF, Ratanasiripong N, Kathalae D. Stress and anxiety management in nursing students: biofeedback and mindfulness meditation. J Nurs Educ. (2015) 54:520-4. doi: 10.3928/01484834-20150814-07

44. Rockstroh C, Blum J, Göritz AS. Combining VR and biofeedback: the effects on perceived restorativeness and presence. J Media Psychol Theor Methods Appl. (2020) 32:176-86. doi: 10.1027/1864-1105/a000270

45. Luijcks R, Hermens HJ, Bodar L, Vossen CJ, Van Os J, Lousberg R. Experimentally induced stress validated by EMG activity. PLoS ONE. (2014) 9:e95215. doi: 10.1371/journal.pone.0095215

46. Rajendra Acharya U, Paul Joseph K, Kannathal N, Lim CM, Suri JS. Heart rate variability: a review. Med Biol Eng Comput. (2006) 44:103151. doi: 10.1007/s11517-006-0119-0

47. Clays E, De Bacquer D, Crasset V, Kittel F, de Smet P, Kornitzer $\mathrm{M}$, et al. The perception of work stressors is related to reduced parasympathetic activity. Int Arch Occup Environ Health. (2011) 84:18591. doi: 10.1007/s00420-010-0537-z

48. Hjortskov N, Rissen D, Blangsted AK, Fallentin N, Lundberg U, Sogaard $\mathrm{K}$. The effect of mental stress on heart rate variability and blood pressure during computer work. Eur J Appl Physiol. (2004) 92:849. doi: 10.1007/s00421-004-1055-z

49. Kaegi DM, Halamek LP, Van Hare GF, Howard SK, Dubin AM. Effect of mental stress on heart rate variability: validation of simulated operating and delivery room training modules. Pediat Res. (1999) 45:77. doi: 10.1203/00006450-199904020-00463

50. Orsila R, Virtanen M, Luukkaala T, Tarvainen M, Karjalainen P, Viik J, et al. Perceived mental stress and reactions in heart rate variability-a pilot study among employees of an electronics company. Int J Occup Saf Ergon. (2008) 14:275-83. doi: 10.1080/10803548.2008.11076767

51. Sloan R, Shapiro P, Bagiella E, Boni S, Paik M, Bigger J Jr, et al. Effect of mental stress throughout the day on cardiac autonomic control. Biol Psychol. (1994) 37:89-99. doi: 10.1016/0301-0511(94)90024-8 
52. Michels N, Sioen I, Clays E, De Buyzere M, Ahrens W, Huybrechts I, et al. Children's heart rate variability as stress indicator: association with reported stress and cortisol. Biol Psychol. (2013) 94:433-40. doi: 10.1016/j.biopsycho.2013. 08.005

53. Cole SR. Assessment of differential item functioning in the Perceived Stress Scale-10. J Epidemiol Community Health. (1999) 53:319-20. doi: 10.1136/jech.53.5.319

54. Sheehan DV, Lecrubier Y, Sheehan KH, Amorim P, Janavs J, Weiller E, et al. The Mini-International Neuropsychiatric Interview (M.I.N.I.): the development and validation of a structured diagnostic psychiatric interview for DSM-IV and ICD-10. J Clin Psychiatry. (1998) 59(Suppl. 20):22-33; quiz 4-57.

55. American Psychiatric Association. Diagnostic and Statistical Manual of Mental Disorders: DSM-5. 5th ed. Washington, DC: American Psychiatric Association (2013). doi: 10.1176/appi.books.9780890425596

56. Otto B, Riepl RL, Klosterhalfen S, Enck P. Endocrine correlates of acute nausea and vomiting. Auton Neurosci. (2006) 129:1721. doi: 10.1016/j.autneu.2006.07.010

57. Xu LH, Koch KL, Summy-Long J, Stern RM, Seaton JF, Harrison TS, et al. Hypothalamic and gastric myoelectrical responses during vectioninduced nausea in healthy Chinese subjects. Am J Physiol. (1993) 265:E57884. doi: 10.1152/ajpendo.1993.265.4.E578

58. Wang J, Rao H, Wetmore GS, Furlan PM, Korczykowski M, Dinges DF, et al. Perfusion functional MRI reveals cerebral blood flow pattern under psychological stress. Proc Nat Acad Sci U. S. A. (2005) 102:178049. doi: 10.1073/pnas.0503082102

59. Bouguet J-Y. Pyramidal Implementation of the Lucas Kanade Feature Tracker. Palo Alto, CA: Open Source Computer Vision Library (2003).

60. Kelly D. Motion and vision. II. Stabilized spatio-temporal threshold surface: Erratum. J Optic Soc Am. (1980) 7:1173.

61. Spielberger CD. State-trait anxiety inventory. In: Weiner IB, Craighead WE, editors. The Corsini Encyclopedia of Psychology. Hoboken, NJ: John Wiley (2010). doi: 10.1002/9780470479216.corpsy0943

62. Paice JA, Cohen FL. Validity of a verbally administered numeric rating scale to measure cancer pain intensity. Cancer Nurs. (1997) 20:8893. doi: 10.1097/00002820-199704000-00002

63. Crawford JR, Henry JD. The positive and negative affect schedule (PANAS): construct validity, measurement properties and normative data in a large non-clinical sample. Br J Clin Psychol. (2004) 43:24565. doi: 10.1348/0144665031752934

64. Leon AC, Olfson M, Portera L, Farber L, Sheehan DV. Assessing psychiatric impairment in primary care with the Sheehan Disability Scale. Int J Psychiatry Med. (1997) 27:93-105. doi: 10.2190/T8EM-C8YH-373N-1UWD

65. Herdman M, Gudex C, Lloyd A, Janssen M, Kind P, Parkin $\mathrm{D}$, et al. Development and preliminary testing of the new fivelevel version of EQ-5D (EQ-5D-5L). Qual Life Res. (2011) 20:1727-36. doi: 10.1007/s11136-011-9903-X

66. Kennedy RS, Lane NE, Berbaum KS, Lilienthal MG. Simulator sickness questionnaire: an enhanced method for quantifying simulator sickness. Int J Aviat Psychol. (1993) 3:203-20. doi: 10.1207/s15327108ijap 0303_3
67. Choi J, Lee KK, Choi J. Determinants of user satisfaction with mobile vr headsets: the human factors approach by the user reviews analysis and product lab testing. Int J Cont. (2019) 15:1-9. doi: 10.5392/IJoC.2019.15.1.001

68. Rebenitsch L, Owen C. Review on cybersickness in applications and visual displays. Virt Real. (2016) 20:101-25. doi: 10.1007/s10055-016-0285-9

69. Shaffer F, Ginsberg JP. An overview of heart rate variability metrics and norms. Front Public Health. (2017) 5:258. doi: 10.3389/fpubh.2017.00258

70. Umetani K, Singer DH, McCraty R, Atkinson M. Twenty-four hour time domain heart rate variability and heart rate: relations to age and gender over nine decades. J Am Coll Cardiol. (1998) 31:593601. doi: 10.1016/S0735-1097(97)00554-8

71. Filaire E, Portier H, Massart A, Ramat L, Teixeira A. Effect of lecturing to 200 students on heart rate variability and alpha-amylase activity. Eur J Appl Physiol. (2010) 108:1035-43. doi: 10.1007/s00421-009-1310-4

72. Shaffer F, McCraty R, Zerr CL. A healthy heart is not a metronome: an integrative review of the heart's anatomy and heart rate variability. Front Psychol. (2014) 5:1040. doi: 10.3389/fpsyg.2014.01040

73. Lucini D, Norbiato G, Clerici M, Pagani M. Hemodynamic and autonomic adjustments to real life stress conditions in humans. Hypertension. (2002) 39:184-8. doi: 10.1161/hy0102.100784

74. Kaplan S. The restorative benefits of nature: toward an integrative framework. J Environ Psychol. (1995) 15:169-82. doi: 10.1016/0272-4944(95)90001-2

75. Valtchanov D, Barton KR, Ellard C. Restorative effects of virtual nature settings. Cyberpsychol Behav Soc Netw. (2010) 13:503-12. doi: 10.1089/cyber.2009.0308

76. Mason JW. A historical view of the stress field. J Human Stress. (1975) 1:22-36. doi: 10.1080/0097840X.1975.9940405

77. Selye H. The evolution of the stress concept. Am Sci. (1973) 61:692-9.

78. Selye H. Confusion and controversy in the stress field. J Human Stress. (1975) 1:37-44. doi: 10.1080/0097840X.1975.9940406

Conflict of Interest: JDKK and DK were employed by Samsung Electronics Co., Ltd.

The remaining authors declare that the research was conducted in the absence of any commercial or financial relationships that could be construed as a potential conflict of interest.

Publisher's Note: All claims expressed in this article are solely those of the authors and do not necessarily represent those of their affiliated organizations, or those of the publisher, the editors and the reviewers. Any product that may be evaluated in this article, or claim that may be made by its manufacturer, is not guaranteed or endorsed by the publisher.

Copyright (c) 2021 Kim, Kim, Kim, Chung, Park, Kim, Kim, Kim, Kim and Jeon. This is an open-access article distributed under the terms of the Creative Commons Attribution License (CC BY). The use, distribution or reproduction in other forums is permitted, provided the original author(s) and the copyright owner(s) are credited and that the original publication in this journal is cited, in accordance with accepted academic practice. No use, distribution or reproduction is permitted which does not comply with these terms. 